\title{
Small-time asymptotics for Implied volatility under the Heston model
}

\author{
Martin Forde ${ }^{* \dagger} \quad$ Antoine Jacquier ${ }^{\ddagger}$
}

\begin{abstract}
${ }^{1}$ We rigourize the work of Lewis[Lewis07] and Durrleman[Durr05] on the small-time asymptotic behaviour of the implied volatility under the Heston stochastic volatility model (Theorem 1.1). We apply the Gärtner-Ellis theorem from large deviations theory to the exponential affine closed-form expression for the moment generating function of the log forward price, to show that it satisfies a small-time large deviation principle. The rate function is computed as Fenchel-Legendre transform, and we show that this can actually be computed as a standard Legendre transform, which is a simple numerical root-finding exercise. We establish the corresponding result for implied volatility in Theorem 2.4, using well known bounds on the standard Normal distribution function. In Theorem 2.5 we compute the level, the slope and the curvature of the implied volatility in the small-maturity money At-the-money, and the answer is consistent with that obtained by formal PDE methods by Lewis[Lewis00] and probabilistic methods by Durrleman[Durr04].
\end{abstract}

\section{Introduction}

In recent years there has been an explosion of literature on small-time asymptotics for stochastic volatility models, see Hagan et al.[HKLW02], Berestycki et al.[BBF02],[BBF04], Henry-Labordère [HL05],[HL07] and Laurence[Laur08]. All these articles are essentially higher order corrections and/or applications of the seminal work of Varadhan[VarI],[VarII], who showed that the smalltime behaviour of a diffusion process can be characterized in terms of an Energy/distance function on a Riemmanian manifold, whose metric is given by the inverse of the diffusion coefficient (see also Dembo\&Zeitouni[DeZ98]).

For a local volatility model, Berestycki,Busca\&Florent[BBF02] showed that the implied volatility in the small-maturity limit is the harmonic mean of the local volatility. For a general stochastic

\footnotetext{
*Department of Mathematical Sciences, Dublin City University, Glasnevin, Dublin 9, Ireland (Martin.Forde@dcu.ie).

†The work of Forde has been supported by an SFI grant for the Edgeworth Centre for Financial Mathematics and the European Science Foundation, AMaMeF Exchange Grant 2107.

$\ddagger$ Imperial College, 180 Queen’s Gate, Department of Mathematics, London and Zeliade Systems, 56 Rue JeanJacques Rousseau, Paris (antoine.jacquier08@imperial.ac.uk).

${ }^{1}$ The authors would like to thank Jin Feng, Jean-Pierre Fouque, Alan Lewis, Roger Lee and Aleksandar Mijatović for many useful discussions.
} 
volatility model satisfying certain growth conditions, Berestycki et al.[BBF04] show that the implied volatility in the small-maturity limit can be characterized as a viscosity solution to a first order non-linear eikonal PDE. Viscosity solutions are a weak formulation for solutions to PDEs introduced by Crandall\&Lions[CL84]. Viscosity solutions can be non-differentiable, but are in some sense sandwiched between a differentiable sub- and super- solution, which we call test functions (see Fleming\&Soner[FS93]). In general, for optimal control problems, the value function is not smooth enough to satisfy the dynamic programming equation in the classical sense, but it is the unique viscosity solution to that equation.

The solution to this eikonal equation is the length of the shortest geodesic from a point to a line under the aforementioned metric i.e. it is a variable endpoint variational problem. Durrleman[Durr05] applied the Legendre transform to the eikonal equation for this shortest geodesic distance under the Heston model, and then solved the resulting ODE. Durrleman did not address the highly non-trivial issue of existence and uniqueness of solutions to this eikonal equation. Lewis[Lewis07] generalized the work of Durrleman, providing a formal derivation of the small-time implied volatility asymptotics for a general $\operatorname{CEV}(p)$-volatility model, which nests the Heston model. Lewis computed the length of the aforementioned geodesics using conserved energy and momentum arguments that arise from integrating the geodesic equations, and a transversality condition for the variable endpoint problem.

Feng,Forde\&Fouque[FFF09] consider the asymptotic behaviour of the Heston model in a regime where the maturity is small, but large compared to the mean reversion time for the volatility process. Using the fact that the Gärtner-Ellis theorem generalizes under Mosco convergence, they establish a Large deviation principle for the log Stock price in this small-time, fast meanreverting regime, and derive corresponding results for call option prices and implied volatility. Medvedev\&Scaillet[MS04] consider a general stochastic volatility model and derive an asymptotic formula for small maturities in terms of a certain modified moneyness; this parametrization does not capture the effect of large deviations because the strike is forced to converge to the spot price as the maturity goes to zero. Alòs\&Ewald[AE08] take a very novel approach, and use Malliavin calculus to derive an approximate pricing formula for European options under the Heston model when the maturity and the volatility-of-variance are $\ll 1$.

In this article, we provide a rigorous analysis of the small-time Heston stochastic volatility model. The Heston model is problematic because the singular coefficient $\sqrt{y}$ in the volatility-ofvariance function is not Lipschitz with respect to the usual Euclidean metric, so we cannot readily apply the standard Freidlin-Wentzell(FW) theory of large deviations for stochastic differential equations. Moreover, it is non-trivial to establish whether the comparison principle holds for the Hamiltonian associated with the Heston process. We require the comparison principle to hold if we wish to apply the extensions of FW theory outlined in Feng\&Kurtz[FK06] to establish a trajectory-level Large deviation principle. In this article we take a simpler route, by analyzing the well known closed-form expression for the moment generating function of the log Stock price for the Heston model, and using the Gärtner-Ellis theorem from large deviations theory to show that the $\log$ forward price satisfies a small-time large deviation principle (Theorem 1.1). In this way, we sidestep the problem of existence/uniqueness of classical or viscosity solutions to the eikonal equation and/or the variable endpoint calculus of variations problem associated with the Heston model. The rate function is computed as a Fenchel-Legendre transform, and we prove that this 
can actually be computed more efficiently as a Legendre transform. Using well known bounds on the standard Normal distribution function, we then convert this result into a statement about the limiting behaviour of the implied volatility of a call option in the small-time regime (Theorem 2.4).

This article is similar in spirit to Feng et al.[FFF09]; in this article the term involving $y_{0}$ in the log Stock price mgf dominates in the small-time regime, but in the [FFF09] regime the asymptotics are dominated by the ergodic behaviour of the variance process which is independent of $y_{0}$.

\section{A small-time Large deviation principle for the log Stock price under the Heston model}

We work on a model $(\Omega, \mathcal{F}, \mathbb{P})$ with a filtration $\left(\mathcal{F}_{t}\right)_{t \geq 0}$ supporting two Brownian motions, and satisfying the usual conditions. All expectations are taken under $\mathbb{P}$ unless otherwise stated.

Theorem 1.1 Consider the Heston stochastic volatility model for a log forward price process $X_{t}$, defined by the following stochastic differential equations

$$
\left\{\begin{array}{l}
d X_{t}=-\frac{1}{2} Y_{t} d t+\sqrt{Y_{t}} d W_{t}^{1} \\
d Y_{t}=\kappa\left(\theta-Y_{t}\right) d t+\sigma \sqrt{Y_{t}} d W_{t}^{2} \\
d W_{t}^{1} d W_{t}^{2}=\rho d t
\end{array}\right.
$$

with $X_{0}=x_{0}, Y_{0}=y_{0}, \kappa, y_{\infty}, \sigma>0,|\rho|<1$ and $2 \kappa y_{\infty}>\sigma^{2}$, so that $Y=0$ is an unattainable barrier, where $W$ and $B$ are two correlated Brownian motions. ${ }^{2}$ Then $X_{t}-x_{0}$ satisfies a Large deviation principle $(L D P)$ as $t \rightarrow 0$, with rate function $\Lambda^{*}(x)$ equal to the Legendre transform of the continuous function $\Lambda: \mathbb{R} \rightarrow \mathbb{R}^{+} \cup\{\infty\}$ given by

$$
\begin{aligned}
\Lambda(p) & =\frac{y_{0} p}{\sigma\left(\sqrt{1-\rho^{2}} \cot \left(\frac{1}{2} \sigma p \sqrt{1-\rho^{2}}\right)-\rho\right)} \text { for } p \in\left(p_{-}, p_{+}\right) \\
& =\infty \text { for } p \notin\left(p_{-}, p_{+}\right)
\end{aligned}
$$

where the following table shows how to compute the values of $p_{-}$and $p_{+}$

\begin{tabular}{|c|c|c|}
\hline$\rho$ & $p_{-}$ & $p_{+}$ \\
\hline$<0$ & $\frac{\arctan \left(\frac{\sqrt{1-\rho^{2}}}{\rho}\right)}{\frac{1}{2} \sigma \sqrt{1-\rho^{2}}}$ & $\frac{\pi+\arctan \left(\frac{\sqrt{1-\rho^{2}}}{\rho}\right)}{\frac{1}{2} \sigma \sqrt{1-\rho^{2}}}$ \\
\hline$=0$ & $-\frac{\pi}{\sigma}$ & $\frac{\pi}{\sigma}$ \\
\hline$>0$ & $\frac{-\pi+\arctan \left(\frac{\sqrt{1-\rho^{2}}}{\rho}\right)}{\frac{1}{2} \sigma \sqrt{1-\rho^{2}}}$ & $\frac{\arctan \left(\frac{\sqrt{1-\rho^{2}}}{\rho}\right)}{\frac{1}{2} \sigma \sqrt{1-\rho^{2}}}$. \\
\hline
\end{tabular}

We also have that

$$
-\lim _{t \rightarrow 0} t \log \mathbb{P}\left(X_{t}-x_{0}>k\right)=-\lim _{t \rightarrow 0} t \log \mathbb{P}\left(X_{t}-x_{0} \geq k\right)=\inf _{\{x: x \geq k\}} \Lambda^{*}(x)=\Lambda^{*}(k)
$$

\footnotetext{
${ }^{2}$ The $Y$ process is the Cox-Ingersoll-Ross (CIR) diffusion (also known as the square root process) and it satisfies the Yamada-Watanabe condition (see page 291, Proposition 2.13 in Karatzas\&Shreve[KS91]), so it admits a unique strong solution. The $X$ process can be expressed as a stochastic integral of the $Y$ process, so the $X$ process is also well defined.
} 
for $k>0$, because $\Lambda^{*}$ is continuous and strictly increasing when $x>0$, and

$$
-\lim _{t \rightarrow 0} t \log \mathbb{P}\left(X_{t}-x_{0}<k\right)=-\lim _{t \rightarrow 0} t \log \mathbb{P}\left(X_{t}-x_{0} \leq k\right)=\inf _{\{x: x \leq k\}} \Lambda^{*^{\prime}}(x)=\Lambda^{*}(k)
$$

for $k<0$, because $\Lambda^{*}$ is continuous and strictly decreasing when $x<0$.

Proof. From Albrecher,Mayer,Schoutens\&Tistaert[AMST06], we have the following closed-form expression for the characteristic function $\phi(k, t)$ of $X_{t}-x_{0}$

$\phi(k, t)=\mathbb{E}\left(e^{i k\left(X_{t}-X_{0}\right)}\right)=\exp \left\{\frac{\kappa \theta}{\sigma^{2}}\left[(\kappa-\rho \sigma i k-d) t-2 \log \left(\frac{1-g_{2} e^{-d t}}{1-g_{2}}\right)\right]\right\} \exp \left\{\frac{y_{0}}{\sigma^{2}}(\kappa-\rho \sigma i k-d) \frac{1-e^{-d t}}{1-g_{2} e^{-d t}}\right\}$,

where

$$
\begin{aligned}
g_{2} & =g_{2}(k)=\frac{\kappa-\rho \sigma i k-d(k)}{\kappa-\rho \sigma i k+d(k)}, \\
d & =d(k)=\sqrt{(\kappa-\rho \sigma i k)^{2}+\sigma^{2}\left(i k+k^{2}\right)},
\end{aligned}
$$

and we take the principal branch for the complex log function. For the square root here, either of the two roots may be chosen, because the characteristic function is even in $d$. We now recall the following theorem

Theorem 1.2 (Lukacs[Lukacs70], Theorem 7.1.1). If a characteristic function $\psi(k)$ is regular ${ }^{3}$ in the neighborhood of $k=0$, then it is also regular in a horizontal strip and can be represented in this strip by a Fourier integral. This strip is either the whole plane, or it has one or two horizontal boundary lines. The purely imaginary points on the boundary of the strip of regularity (if this strip is not the whole plane) are singular points of $\psi(z)$.

(see also Lewis[Lewis00]). $\kappa>0$ by assumption, so the square root function $\sqrt{z}$ that appears in the definition of $d($.$) is analytic at z=\kappa^{2}$. Also, for $t$ sufficiently small and $k=0$, the argument being passed to the complex logarithm function in Eq 5 tends to 1 where the log function is analytic. Thus, for $t$ sufficiently small, the characteristic function of $X_{t}-x_{0}$ is analytic at $k=0$, so substituting $k=-i p$ for $p \in \mathbb{R}$ in $\mathrm{Eq} 5$, we can apply Theorem 1.2 to obtain the analytic continuation

$$
\begin{aligned}
\mathbb{E}\left(e^{p\left(X_{t}-x_{0}\right)}\right) & =\exp \left\{C(t, p)+D(t, p) y_{0}\right\} \text { for } t<T^{*}(p) \\
& =+\infty \text { for } t \geq T^{*}(p),
\end{aligned}
$$

where

$$
\begin{aligned}
C(t, p) & =\frac{\kappa \theta}{\sigma^{2}}\left[(\kappa-\rho \sigma p-d) t-2 \log \left(\frac{1-g_{2} e^{-d t}}{1-g_{2}}\right)\right], \\
D(t, p) & =\frac{y_{0}}{\sigma^{2}}(\kappa-\rho \sigma p-d) \frac{1-e^{-d t}}{1-g_{2} e^{-d t}}, \\
g_{2} & =g_{2}(p)=\frac{\kappa-\rho \sigma p-d(p)}{\kappa-\rho \sigma p+d(p)}, \\
d & =d(p)=\sqrt{(\kappa-\rho \sigma p)^{2}+\sigma^{2}\left(p-p^{2}\right)}
\end{aligned}
$$

\footnotetext{
${ }^{3} \mathrm{~A}$ characteristic function $\psi(u)$ is regular if it is analytic and single-valued. $\psi(u)$ is analytic if there exists a function $A: \mathbb{C} \rightarrow \mathbb{C}$ which is analytic in some circle $z:|z|<r$, and $A(z)=\psi(z)$ for $z \in \mathbb{R}$ in a neighborhood of $k=0$ on the real line.
} 
and

$$
T^{*}(p)=\frac{2}{\sqrt{\sigma^{2} p(p-1)-(\kappa-\rho \sigma p)^{2}}}\left[\pi 1_{\{\rho \sigma p-\kappa<0\}}+\arctan \left(\frac{\sqrt{\sigma^{2} p(p-1)-(\kappa-\rho \sigma p)^{2}}}{\rho \sigma p-\kappa}\right)\right]
$$

is the critical explosion time for the $p$ th moment if

$$
\sigma^{2} p(p-1)>(\kappa-\rho \sigma p)^{2}
$$

(the expression for $T^{*}(p)$ comes from Proposition 3.1, part (iii) in Andersen\&Piterbarg[AP07], see also Keller-Ressel[KR08]). The moment explosion is caused by $\bar{b} \cot \left(\frac{1}{2} \bar{b} t\right)+b$ tending to zero as $t \searrow 0$. Setting $p \mapsto \frac{p}{t}$ and letting $t \searrow 0$ in Eq 10, we see that condition 11 is satisfied for $t$ sufficiently small, and we have

$$
\begin{aligned}
T^{*}\left(\frac{p}{t}\right) & \sim \frac{2 t}{\sigma|p| \sqrt{1-\rho^{2}}}\left[\pi 1_{\{\rho p \leq 0\}}+\operatorname{sgn}(p) \arctan \left(\frac{\sqrt{1-\rho^{2}}}{\rho}\right)\right] \text { as } t \rightarrow 0 \text { for } \rho \neq 0, p \neq 0 \\
& \sim \frac{\pi t}{\sigma|p|} \text { as } t \rightarrow 0 \text { for } \rho=0, p \neq 0 \\
& =\infty \text { for } p=0
\end{aligned}
$$

where we have used the fact that $\arctan (x) \rightarrow-\frac{1}{2} \pi$ as $x \rightarrow-\infty$ to deal with the case $\rho=0, p \neq 0$ (also note that we are not letting $\rho \rightarrow 0$ tend to zero here, but rather we are fixing $\rho=0$ and letting $t \rightarrow 0$. By careful examination of the entries of Table 1.1 we see that for any $p \in\left(p_{-}, p_{+}\right)$ we have $T^{*}\left(\frac{p}{t}\right)>t$ for $t$ sufficiently small, so Eq 7 is valid. Letting $t \searrow 0$ and $\theta=\sigma \bar{\rho} p$, we find that

$$
\begin{aligned}
d\left(\frac{p}{t}\right) & \sim \frac{i \sigma \bar{\rho} p}{t}, \\
g_{2}\left(\frac{p}{t}\right) & \sim \frac{\rho+i \bar{\rho}}{\rho-i \bar{\rho}}, \\
t y_{0} D\left(\frac{p}{t}\right) & \sim-\frac{y_{0} p}{\sigma^{2}}(\rho \sigma+i \sigma \bar{\rho}) \cdot \frac{1-e^{-i \sigma \bar{\rho} p}}{1-\frac{\rho+i \bar{\rho}}{\rho-i \bar{\rho}} e^{-i \sigma \bar{\rho} p}}, \\
& =-\frac{y_{0} p}{\sigma}(\rho+i \bar{\rho}) \cdot \frac{(\rho-i \bar{\rho})\left(e^{i \theta / 2}-e^{-i \theta / 2}\right)}{(\rho-i \bar{\rho}) e^{i \theta / 2}-(\rho+i \bar{\rho}) e^{-i \theta / 2}} \\
& =-\frac{y_{0} p}{\sigma} \cdot \frac{(\rho+i \bar{\rho})(\rho-i \bar{\rho})\left(e^{i \theta / 2}-e^{-i \theta / 2}\right)}{\rho\left(e^{i \theta / 2}-e^{-i \theta / 2}\right)-i \bar{\rho}\left(e^{i \theta / 2}+e^{-i \theta / 2}\right)} \\
& =\frac{y_{0} p}{\sigma} \cdot \frac{1}{i \bar{\rho} \operatorname{coth}\left(\frac{1}{2} i \theta\right)-\rho} \\
& =\Lambda(p), \\
t C\left(\frac{p}{t}, p\right) & \sim t \frac{\kappa \theta}{\sigma^{2}}\left[(-\rho-i \bar{\rho}) \sigma p-2 \log \left(\frac{1-\frac{\rho+i \bar{\rho}}{\rho-i \bar{\rho}} e^{-i \theta}}{1-\frac{\rho+i \bar{\rho}}{\rho-i \bar{\rho}}}\right)\right]
\end{aligned}
$$

where $\bar{\rho}=\sqrt{1-\rho^{2}}$. Thus

$$
\lim _{t \rightarrow 0} t \log \mathbb{E}\left(e^{\frac{p}{t}\left(X_{t}-x_{0}\right)}\right)=\Lambda(p)
$$


for $p \in\left(p_{-}, p_{+}\right)$(see Table 1.1 for a definition of $p_{-}$and $p_{+}$), where $\Lambda($.$) is defined in Eq 2. By$ direct verification, we see that $\Lambda(p) \rightarrow \infty$ as $p \nearrow p_{+}$and as $p \searrow p_{-}$. We also need to verify that $\lim _{t \rightarrow 0} t \log \mathbb{E}\left(e^{\frac{p}{t}\left(X_{t}-x_{0}\right)}\right)=\infty$ for $p \notin\left(p_{-}, p_{+}\right)$. But this follows from the monotonicity result in Lemma B.1 when we let $t \rightarrow 0$. Thus Assumption A.1 is satisfied. $\Lambda$ is smooth in $\left(p_{-}, p_{+}\right)$and

$$
\begin{aligned}
\Lambda^{\prime}(p) & =\frac{y_{0}}{\sigma\left(\sqrt{1-\rho^{2}} \cot \frac{\theta}{2}-\rho\right)}+\frac{\sigma y_{0} p\left(1-\rho^{2}\right) \csc ^{2} \frac{\theta}{2}}{2 \sigma\left(\sqrt{1-\rho^{2}} \cot \frac{\theta}{2}-\rho\right)^{2}}, \\
\Lambda^{\prime \prime}(p) & =\frac{y_{0}\left(1-\rho^{2}\right) \csc ^{2} \frac{\theta}{2}\left(1-\frac{\theta}{2} \cot \frac{\theta}{2}\right)}{\left(\sqrt{1-\rho^{2}} \cot \frac{\theta}{2}-\rho\right)^{2}}+\frac{y_{0} \theta\left(1-\rho^{2}\right)^{\frac{3}{2}} \csc ^{4} \frac{\theta}{2}}{2\left(\sqrt{1-\rho^{2}} \cot \frac{\theta}{2}-\rho\right)^{3}} .
\end{aligned}
$$

The first term in Eq 15 is non-negative on $\left(p_{-}, p_{+}\right)$because $\frac{\theta}{2} \cot \frac{\theta}{2} \leq 1$ if $\theta$ in $(-2 \pi, 2 \pi)$, which will be the case when $p$ lies in the interval $\left(p_{-}, p_{+}\right)$(by careful checking of the entries in the table in Theorem 1.1). The second term is also non-negative because it is a non-negative multiple of $\Lambda(p)$ itself which is non-negative, so $\Lambda^{\prime \prime}(p)>0$ for $p \in\left(p_{-}, p_{+}\right)$. By direct inspection, we also have that $\Lambda(p) \rightarrow \infty$ and $\left|\Lambda^{\prime}(p)\right| \rightarrow \infty$ as $p \nearrow p_{+}$and $p \searrow p_{-}$. Thus $\Lambda(p)$ is convex, essentially smooth and lower semi-continuous, so (by the Gärtner-Ellis Theorem in Theorem A.1), $X_{t}-x_{0}$ satisfies the Large deviation principle with rate function equal to the Fenchel-Legendre transform of $\Lambda(p)$. By the essential smoothness property of $\Lambda(p)$, the equation

$$
\left.\frac{\partial}{\partial p}(p x-\Lambda(p))\right|_{p^{*}}=0
$$

has a solution $p^{*}=p^{*}(x)$ in $\left(p_{-}, p_{+}\right)$, which equivalently solves

$$
x=\Lambda^{\prime}\left(p^{*}\right) .
$$

But $\Lambda^{\prime}$ is continuous and strictly monotonically increasing on $\left(p_{-}, p_{+}\right)$, so there is a unique $p^{*}(x)$. The unique minimum $x^{*}$ of $\Lambda^{*}$ occurs at $x^{*}=\left(\Lambda^{*^{\prime}}\right)^{-1}(0)=\Lambda^{\prime}(0)=0$, and $\Lambda^{*}(0)=0$. For $p \in\left(p_{-}, p_{+}\right), \Lambda^{\prime}(p)$ is negative when $p<0$, and is positive when $p>0$. By a convex analysis result , $x=\Lambda^{\prime}(p)$ for $p \in\left(p_{-}, p_{+}\right)$) if and only if $p=\Lambda^{*^{\prime}}(x)$ (see the proof of Lemma 2.5 in Feng et al.[FFF09] for a very similar analysis). Consequently, $\Lambda^{*}$ is strictly increasing when $x>0$ and strictly decreasing when $x<0$, and Eqs 3 and 4 follow .

Remark 1.1 Note that $\Lambda(p)$ does not depend on the drift terms $\kappa$ or $y_{\infty}$, and this is typical in the Freidlin-Wentzell theory of small-time large deviations for diffusion processes.

\section{Small-time behaviour of Call options and Implied volatil- ity}

Two useful corollaries of Theorem 1.1 are the following rare event estimates for pricing out-of-themoney call and put options of small maturity.

Corollary 2.1 We have the following small-time behaviour for out-of-the-money call options on $S_{t}=e^{X_{t}}$

$$
-\lim _{t \rightarrow 0} t \log \mathbb{E}\left(S_{t}-K\right)^{+}=\Lambda^{*}(x),
$$

where $x=\log \left(\frac{K}{S_{0}}\right)>0$ is the log-moneyness. 
Corollary 2.2 We have the following small-time behaviour for out-of-the-money put options on $S_{t}=e^{X_{t}}$

$$
-\lim _{t \rightarrow 0} t \log \mathbb{E}\left(K-S_{t}\right)^{+}=\Lambda^{*}(x),
$$

for $x=\log \left(\frac{K}{S_{0}}\right)<0$.

Proof. (of Corollary 2.1)

(i) We first deal with the lower bound. From a drawing, we see that for any $\delta>0$, we have

$$
\mathbb{E}\left(S_{t}-K\right)^{+} \geq \delta \mathbb{P}\left(S_{t}>K+\delta\right) .
$$

Then by Theorem 1.1 we have that

$$
\liminf _{t \rightarrow 0} t \log \mathbb{E}\left(S_{t}-K\right)^{+} \geq \liminf _{t \rightarrow 0}\left[t \log \delta+t \log \mathbb{P}\left(S_{t}>K+\delta\right)\right] \geq-\Lambda^{*}\left(\log \frac{K+\delta}{S_{0}}\right) .
$$

Take $\delta \rightarrow 0+$. By continuity of $\Lambda^{*}(x)$, we have the desired lower bound.

(ii) To obtain the desired upper bound, we note (by Hölder's inequality) that for any $p, q>1$ with $p^{-1}+q^{-1}=1$, we have

$$
\begin{aligned}
\mathbb{E}\left(S_{t}-K\right)^{+} & =\mathbb{E}\left[\left(S_{t}-K\right)^{+} 1_{\left\{S_{t} \geq K\right\}}\right] \leq\left[\mathbb{E}\left(\left(S_{t}-K\right)^{+}\right)^{p}\right]^{1 / p} \mathbb{E}\left(1_{\left\{S_{t} \geq K\right\}}^{q}\right)^{1 / q} \\
& =\left[\mathbb{E}\left(\left(S_{t}-K\right)^{+}\right)^{p}\right]^{1 / p} \mathbb{P}\left(S_{t} \geq K\right)^{1 / q} . \\
& \leq\left[\mathbb{E}\left(S_{t}^{p}\right)\right]^{1 / p} \mathbb{P}\left(S_{t} \geq K\right)^{1-1 / p} .
\end{aligned}
$$

Taking logs and multiplying by $t$ we obtain

$$
t \log \mathbb{E}\left(S_{t}-K\right)^{+} \leq \frac{t}{p} \log \mathbb{E}\left(S_{t}^{p}\right)+t\left(1-\frac{1}{p}\right) \log \mathbb{P}\left(S_{t} \geq K\right) .
$$

We claim that $t \log \mathbb{E}\left(S_{t}^{p}\right) \rightarrow 0$ as $t \rightarrow 0$. Consider $\delta>0$. For $t$ sufficiently small, $\frac{\delta}{t}>p$. Then we have

$$
\limsup _{t \rightarrow 0} t \log \mathbb{E}\left(\left(\frac{S_{t}}{S_{0}}\right)^{p}\right) \leq \limsup _{t \rightarrow 0} t \log \mathbb{E}\left(\left(\frac{S_{t}}{S_{0}}\right)^{\delta / t}\right)=\Lambda(\delta),
$$

by Lemma B.1, and

$$
\liminf _{t \rightarrow 0} t \log \mathbb{E}\left(\left(\frac{S_{t}}{S_{0}}\right)^{p}\right) \geq \liminf _{t \rightarrow 0} t \log \left(\mathbb{E}\left(\frac{S_{t}}{S_{0}}\right)\right)^{p}=0=\Lambda(0)
$$

by Jensen's inequality. But $\Lambda$ is continuous, so the claim is verified. If we then take $\lim _{p \rightarrow \infty}$ on both sides of Eq 24, we have (by Theorem 1.1) the upper bound

$$
\limsup _{t \rightarrow 0} t \log \mathbb{E}\left(S_{t}-K\right)^{+} \leq-\Lambda^{*}\left(\log \frac{K}{S_{0}}\right) .
$$

The proof of Corollary 2.2 follows by a similar argument.

Using the put-call parity, we can combine Corollaries 2.1 and 2.2 to obtain the following result, which holds for all $x \in \mathbb{R}, x \neq 0$. 


\section{Corollary 2.3}

$$
t \log \left(\mathbb{E}\left(S_{t}-K\right)^{+}-\left(S_{0}-K\right)^{+}\right)=t \log \left(\mathbb{E}\left(K-S_{T}\right)^{+}-\left(K-S_{0}\right)^{+}\right)=\Lambda^{*}(x),
$$

where $x=\log \frac{K}{S_{0}}$.

We can also compute the asymptotic implied volatility as follows

Theorem 2.4 We have the following asymptotic behaviour for the implied volatility $\sigma_{t}=\sigma_{t}(x)$ of a European call option on $S_{t}=e^{X_{t}}$, with strike $K=S_{0} e^{x}(x \in \mathbb{R}), x \neq 0$, as $t \rightarrow 0$

$$
I(x)=\lim _{t \rightarrow 0} \sigma_{t}(x)=\frac{x}{\sqrt{2 \Lambda^{*}(x)}} .
$$

Proof. We first assume that $x>0$. We first establish the lower bound for $\sigma_{t}(x)$. Let $n(z)=$ $\frac{1}{\sqrt{2 \pi}} e^{-\frac{1}{2} z^{2}}$, and $\Phi(z)=\int_{-\infty}^{z} n(u) d u$ denote the standard cumulative Normal distribution function. Using the classical notation for the Black-Scholes formula, we set $d_{1}=\frac{-x+\frac{1}{2} \sigma_{t}^{2} t}{\sigma_{t} \sqrt{t}}, d_{2}=d_{1}-\sigma_{t} \sqrt{t}$. By Corollary 2.1 and the definition of implied volatility, we know that for all $\delta, C>0$, there exists a $t^{*}=t^{*}(\delta)>0$ such that for all $t<t^{*}$ we have

$$
\begin{aligned}
e^{-\left(\Lambda^{*}(x)+\delta\right) / t} & \leq \mathbb{E}\left(S_{t}-K\right)^{+} \\
& =S_{0} \Phi\left(d_{1}\right)-S_{0} e^{x} \Phi\left(d_{2}\right) \\
& \leq S_{0} \Phi^{c}\left(-d_{1}\right) \\
& \leq S_{0} \cdot \frac{1}{\left|d_{1}\right|} n\left(d_{1}\right) \\
& \leq S_{0} C \cdot n\left(d_{1}\right),
\end{aligned}
$$

The last two lines follow from the standard estimate on the normal distribution in section 14.8 of Williams[Will91] (see Appendix C), and the fact that the dimensionless implied variance $\sigma_{t}^{2} t \rightarrow 0$ as $t \rightarrow 0$, because the call price tends to zero as $t \rightarrow 0$. Taking logs of both sides and multiplying by $t$, we can find a $t^{* *}=t^{* *}(\delta) \leq t^{*}$ such that for all $t<t^{* *}$ we have

$$
-\left(\Lambda^{*}(x)+\delta\right) \leq t \log \left(S_{0} C\right)-\frac{t}{2} \log (2 \pi)-\frac{\left(x-\frac{1}{2} \sigma_{t}^{2} t\right)^{2}}{2 \sigma_{t}^{2}} \leq-\frac{x^{2}}{2 \sigma_{t}^{2}}+\delta,
$$

and the lower bound follows. For the upper bound, consider $\delta>0$. Again, by Corollary 2.1, there exists an $t(\delta)>0$ such that for all $t<t(\delta)$ we have

$$
\begin{aligned}
e^{-\left(\Lambda^{*}(x)-\delta\right) / t} & \geq \mathbb{E}\left(S_{t}-K\right)^{+} \\
& =S_{0} \Phi\left(d_{1}\right)-S_{0} e^{x} \Phi\left(d_{2}\right) \\
& =S_{0} \Phi^{c}\left(-d_{1}\right)-S_{0} e^{x} \Phi^{c}\left(-d_{2}\right) .
\end{aligned}
$$

Set $d_{2, \delta}=\frac{-x-\delta-\frac{1}{2} \sigma_{t}^{2} t}{\sigma_{t} \sqrt{t}}$. Similar to Corollary 2.1 or by drawing a picture, we can now use the fact that one call option of strike $K$ is worth more than $K\left(e^{\delta}-1\right)$ digital call options of strike $K e^{\delta}$, 
and invoke the lower bound on the Normal distribution in Appendix $\mathrm{C}$ and the fact that $\sigma_{t}^{2} t \rightarrow 0$ as $t \rightarrow 0$ to see that

$$
\begin{aligned}
e^{-\left(\Lambda^{*}(x)-\delta\right) / t} & \geq K\left(e^{\delta}-1\right) \Phi^{c}\left(-d_{2, \delta}\right) \\
& \geq K\left(e^{\delta}-1\right) n\left(d_{2, \delta}\right)\left(\frac{1}{\left|d_{2, \delta}\right|}+\left|d_{2, \delta}\right|\right)^{-1} \\
& \geq K\left(e^{\delta}-1\right) n\left(d_{2, \delta}\right) \cdot \frac{\sigma_{t} \sqrt{t}}{x+\delta}(1-\delta) \\
& \geq K\left(e^{\delta}-1\right) n\left(d_{2, \delta}\right) \cdot e^{-\delta \frac{x+\delta}{\sigma_{t} \sqrt{t}}}(1-\delta) .
\end{aligned}
$$

Taking $\operatorname{logs}$ of both sides and multiplying by $t$, we can further find a $t^{* *} \leq t^{*}$ such that for all $t<t^{* *}$ we have

$$
\begin{aligned}
-\left(\Lambda^{*}(x)-\delta\right) & \geq t \log (K)+t \log \left(e^{\delta}-1\right)-\frac{t}{2} \log (2 \pi)-\frac{\left(x+\delta+\frac{1}{2} \sigma_{t}^{2} t\right)^{2}}{2 \sigma_{t}^{2}}-\sqrt{t} \delta \frac{(x+\delta)}{\sigma_{t}}+t \log (1-\delta) \\
& \geq-\frac{(x+\delta)^{2}}{2 \sigma_{t}^{2}}-\delta .
\end{aligned}
$$

We proceed similarly for $x<0$. 

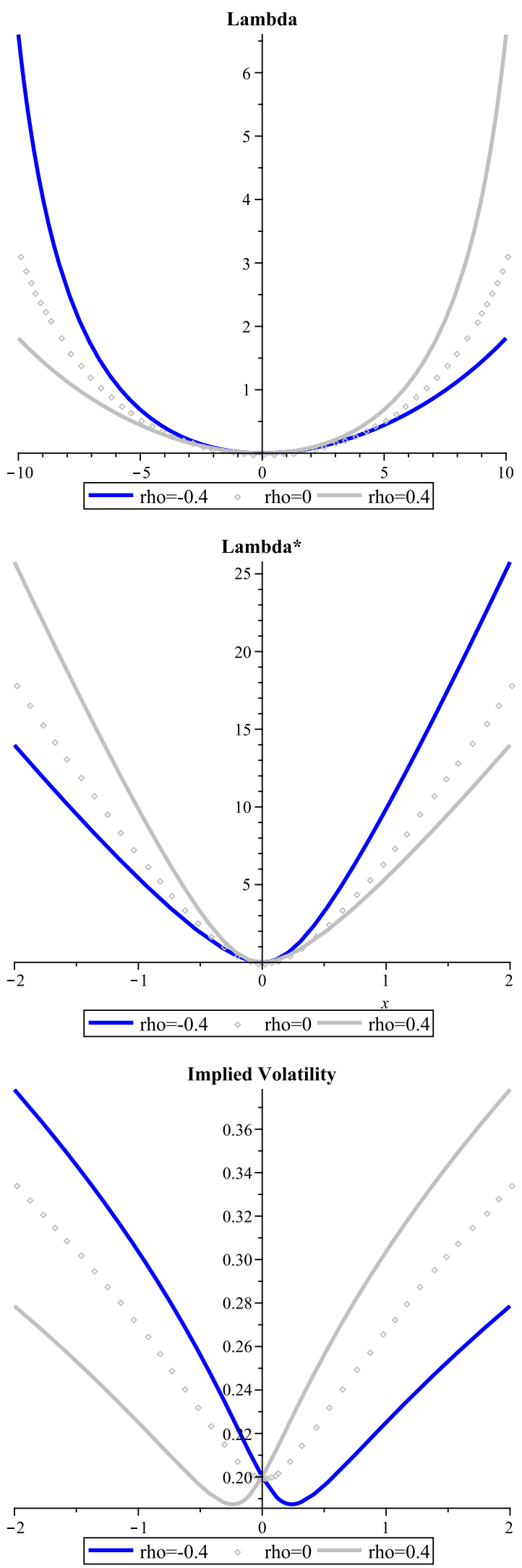

Figure 1: Here we have plotted $\Lambda(p)$, the rate function $\Lambda^{*}(x)$ and the asymptotic implied volatility $I(x)$ for the Heston model with the parameters $y_{0}=.04=y_{\infty}=.04, \sigma=.20$ and $\rho=-0.4,0.0 .4$. We have used the bisection method to numerically compute the Legendre transform $\Lambda^{*}(x)$. 


\subsection{The level, slope and curvature of the small-time implied volatility} At-the-Money

Note that Corollary 2.3 does not deal with the At-the-money case $x=0$. To deal with this case, we use the following Theorem.

Theorem 2.5 The asymptotic implied volatility $I(x)$ has the following expansion around $x=0$

$$
I(x)=\sqrt{y_{0}}\left[1+\frac{1}{4} \rho z+\left(\frac{1}{24}-\frac{5}{48} \rho^{2}\right) z^{2}+O\left(z^{3}\right)\right],
$$

where $z=\frac{\sigma x}{y_{0}}$.

Remark 2.1 Note that $I^{\prime \prime}(0)<0$ for $\rho^{2}>\frac{2}{5}$.

Proof. $\Lambda^{\prime}$ is a diffeomorphism on $\left(p_{-}, p_{+}\right)$and near $p=0$, we have

$$
\begin{aligned}
\Lambda(p) & =\frac{1}{2} y_{0} p^{2}+\frac{1}{4} y_{0} \rho \sigma p^{3}+\frac{1}{24} y_{0} \sigma^{2}\left(1+2 \rho^{2}\right) p^{4}+O\left(p^{5}\right), \\
\Lambda^{\prime}(p) & =y_{0} p+\frac{3}{4} y_{0} \rho \sigma p^{2}+\frac{1}{6} y_{0} \sigma^{2}\left(1+2 \rho^{2}\right) p^{3}+O\left(p^{4}\right) .
\end{aligned}
$$

Recall the following expressions for the first and second derivatives of the inverse of a smooth, invertible function $y(x)$

$$
\begin{aligned}
\frac{d y}{d x} & =\frac{1}{\frac{d x}{d y}} \\
\frac{d^{2} y}{d x^{2}} & =-\frac{d^{2} x}{d y^{2}}\left(\frac{d y}{d x}\right)^{3}
\end{aligned}
$$

Using these equations and setting $x=\Lambda^{\prime}\left(p^{*}(x)\right)$, we find that 


$$
\begin{aligned}
& p^{*^{\prime}}(0)=\frac{1}{\Lambda^{\prime \prime}\left(\Lambda^{\prime-1}(0)\right)}=\frac{1}{\Lambda^{\prime \prime}(0)}=\frac{1}{y_{0}}, \\
& p^{*^{\prime \prime}}(0)=-\frac{\Lambda^{\prime \prime \prime}(0)}{\left(\Lambda^{\prime \prime}(0)\right)^{3}}=-\frac{3 \rho \sigma}{2 y_{0}^{2}} .
\end{aligned}
$$

Thus

$$
p^{*}(x)=\frac{1}{y_{0}} x-\frac{3 \rho \sigma}{4 y_{0}^{2}} x^{2}+O\left(x^{3}\right)
$$

and

$$
I(x)=\frac{x}{\sqrt{2 \Lambda^{*}(x)}}=\frac{1}{\sqrt{2\left(p^{*}(x) x-\Lambda\left(p^{*}(x)\right)\right)}}=\sqrt{y_{0}}\left[1+\frac{1}{4} \rho z+\left(\frac{1}{24}-\frac{5}{48} \rho^{2}\right) z^{2}+O\left(z^{3}\right)\right]
$$

Remark 2.2 The $O\left(x^{3}\right)$ term for $p^{*}(x)$ does not affect the final answer for $I(x)$ up to $O\left(z^{3}\right)$.

Remark 2.3 If we set $V(x)=I(x)^{2}$, then

$$
V(x)=y_{0}\left[1+\frac{1}{2} \rho z+\left(\frac{1}{12}-\frac{7}{48} z^{2}\right)+O\left(z^{3}\right)\right]
$$

and this agrees with the formula on page 127 in Lewis[Lewis00], and section 3.1.2. in Durrleman[Durr04].

\section{References}

[AE08] E.Alòs and C.O.Ewald (2008), "Malliavin differentiability of the Heston volatility and applications to option pricing", Advances in Applied Probability, 40(1), 144-62.

[AMST06] Albrecher H., P. Mayer, W. Schoutens, J. Tistaert (2006), "The Little Heston Trap", working paper.

[AP07] Andersen, L.B.G., and V.V.Piterbarg (2007), "Moment Explosions in Stochastic Volatility Models", Finance and Stochastics.

[BCC97] Bakshi, G., C. Cao and Z. Chen, (1997), "Empirical Performance of Alternative Option Pricing Models", Journal of Finance 52, No. 5, 2003-2049.

[BBF02] Berestycki H., J. Busca and I. Florent (2002) "Asymptotics and calibration of local volatility models" Quantitative Finance Volume 2, 61-69.

[BBF04] Berestycki H., J. Busca and I. Florent (2004) "Computing the Implied Volatility in Stochastic Volatility models" Communications on Pure and Applied Mathematics, Vol LVII 1352-1373. 
[CGMY03] Carr, P., H.Geman, D.Madan and M.Yor (2003), "Stochastic volatility for Lévy processes", Mathematical Finance, Vol. 13 No. 3, pp. 345-382.

[CL84] Crandall,M.G. and P.L.Lions (1984), "Viscosity solutions of Hamilton-Jacobi equations", Trans AMS, 277, 1-42.

[DeZ98] Dembo, A. and O.Zeitouni (1998), "Large deviation techniques and applications", Jones and Bartlet publishers, Boston.

[Durr04] Durrleman, V. (2004), "From implied to spot volatilities", PhD dissertation, Princeton University.

[Durr05] Durrleman, V. (2005), "From implied to spot volatilities", working paper, April 2005 version.

[FFF09] Feng, J., M.Forde and J.P.Fouque (2009), "Short maturity asymptotics for a fast meanreverting Heston stochastic volatility model", submitted.

[FS93] Fleming, W.H. and H. Mete Soner (1993), "Controlled Markov processes and viscosity solutions", Springer-Verlag, New York.

[FK06] Feng, J. and T.G.Kurtz (2006), "Large Deviation for Stochastic Processes Mathematical Surveys and Monographs", Vol 131, American Mathematical Society.

[Forde08] Forde, M. (2008), "Small-time asymptotics for a general stochastic volatility model, using Freidlin-Wentzell theory and differential geometry", Vol 131, American Mathematical Society.

[HKLW02] Hagan P., D.Kumar, A.S.Lesniewski and D.E.Woodward (2002), "Managing Smile Risk", Wilmott Magazine.

[HL05] Henry-Labordère, P..(2005), "A General Asymptotic Implied Volatility for Stochastic Volatility Models", working paper.

[HL07] Henry-Labordère, P.(2007), "Combining the SABR and LMM models", Risk.

[KS91] Karatzas, I. and S.Shreve (1991), "Brownian motion and Stochastic Calculus", SpringerVerlag.

[KR08] Keller-Ressel,M. (2008) "Moment Explosions and Long-Term Behavior of Affine Stochastic Volatility Models", forthcoming in Mathematical Finance.

[Laur08] Laurence, P. (2008) "Implied Volatility, Fundamental solutions, asymptotic analysis and symmetry methods", presentation at Linz, Ricam kick-off Workshop.

[LK08] Lord, R. and C.Kahl (2008), "Complex Logarithms in Heston-Like Models", working paper.

[Lewis00] Lewis, A. (2000), "Option valuation under stochastic volatility", Finance Press.

[Lewis07] Lewis, A. (2007), "Geometries and smile asymptotics for a class of Stochastic Volatility models", www.optioncity.net. 
[Lukacs70] Lukacs E. (1970), "Characteristic functions", second ed., Griffin, London.

[MS04] Medvedev, A. \& O. Scaillet (2004), "A Simple Calibration Procedure of Stochastic Volatility Models with Jumps by Short Term Asymptotic", FAME Research Paper Series rp93, International Center for Financial Asset Management and Engineering.

[SZ99] Schöbel, R. and J. Zhu (1999), "Stochastic volatility with an Ornstein-Uhlenbeck process: An extension", European Finance Review 3, 2346.

[VarI] Varadhan, S.R.S. (1967), "On the behavior of the fundamental solution of the heat equation with variable coefficients". Comm. Pure Appl. Math., 20, 431-455.

[VarII] Varadhan S.R.S. (1967), "Diffusion processes in a small time interval", Communications in pure and applied mathematics 20, 659-685.

[Will91] (1991), "Probability with Martingales", Williams, D. (Cambridge Mathematical Textbooks).

\section{APPENDIX}

\section{A The Gärtner-Ellis theorem}

Consider a sequence of random variables $X_{n} \in \mathbb{R}^{d}$, where $X_{n}$ possesses the law $\mu_{n}$ and logarithmic moment generating function

$$
\Lambda_{n}(\lambda)=\log \mathbb{E}\left(e^{\left\langle\lambda, X_{n}\right\rangle}\right) .
$$

Definition 1 (Definition 2.3.5 in Dembo\&Zeitouni[DeZ98]).

Let $\mathcal{D}_{\Lambda}=\left\{\lambda \in \mathbb{R}^{d}: \Lambda(\lambda)<\infty\right\}$. A convex function $\Lambda: \mathbb{R}^{d} \rightarrow(-\infty, \infty]$ is said to be essentially smooth if

- The interior $\mathcal{D}_{\Lambda}^{0}$ of $\mathcal{D}_{\Lambda}$ is non-empty.

- $\Lambda($.$) is differentiable throughout \mathcal{D}_{\Lambda}^{0}$.

- $\Lambda($.$) is steep, namely \lim _{n \rightarrow \infty}\left|\nabla \Lambda\left(\lambda_{n}\right)\right|=\infty$ whenever $\left\{\lambda_{n}\right\}$ is a sequence in $\mathcal{D}_{\Lambda}^{0}$ converging to a boundary point of $\mathcal{D}_{\lambda}^{o}$.

Assumption A.1 (Assumption 2.3.2 in Dembo\&Zeitouni[DeZ98]).

For each $\lambda \in \mathbb{R}^{d}$, we assume that the logarithmic moment generating function, defined as the limit

$$
\Lambda(\lambda)=\lim _{n \rightarrow \infty} \frac{1}{n} \Lambda_{n}(n \lambda)
$$

exists as an extended real number. Further, the origin belongs to the interior of $\mathcal{D}=\left\{\lambda \in \mathbb{R}^{d}\right.$ : $\Lambda(\lambda)<\infty\}$.

We now recall the Gärtner-Ellis theorem: 
Theorem A.1 (see Theorem 2.3.6 in Dembo\&Zeitouni[DeZ987).

Let Assumption 1.1 hold. Then if $\Lambda(\lambda)$ is essentially smooth and lower semicontinuous, the sequence of random variables $X_{n}$ satisfies the Large deviation principle with rate function $\Lambda^{*}(x)$, which is the Fenchel-Legendre transform of $F$, defined by the variational formula

$$
\Lambda^{*}(x)=\sup _{\lambda \in \mathbb{R}}(\lambda x-\Lambda(\lambda)) .
$$

Lemma A.2 (Lemma 2.3.9 (a) in Dembo\&Zeitouni[DeZ98]).

Let Assumption 1.1 hold. Then $\Lambda$ is a convex function, $\Lambda(\lambda)>-\infty$ everywhere, and $\Lambda^{*}(x)$ is a good convex rate function. In one dimension, this means that $\Lambda^{*}(x)$ is non-decreasing in the region $x>0$.

Remark A.1 Since $\mu_{n}=1$, it is necessary that $\inf _{x} I(x)=0$. If $\Lambda^{*}(x)$ is a good rate function, then the level set $\left\{x: \Lambda^{*}(x) \leq \alpha\right\}$ is compact, so we know that the infimum is attained on this compact set because $\Lambda^{*}$ is lower semicontinuous, i.e. there exists at least one point $x^{*}$ for which $\Lambda^{*}\left(x^{*}\right)=0$ (see pages 5 and 6 in Dembo\&ZZeitouni[DeZ98]).

\section{B Monotonicity of the logarithmic transform}

Lemma B.1 Let $S_{t}=e^{X_{t}}$ be a non-negative martingale with $S_{0}=1$, and let $\left|p_{1}\right|<\left|p_{2}\right|$ with $\operatorname{sgn}\left(p_{1}\right)=\operatorname{sgn}\left(p_{2}\right)$. Then

$$
\mathbb{E}\left(e^{\frac{p_{1}}{t} X_{t}}\right) \leq \mathbb{E}\left(e^{\frac{p_{2}}{t} X_{t}}\right)
$$

for $t$ sufficiently small.

Proof. (i) We first consider $0<p_{1}<p_{2}<\infty$. Let $S_{t}=e^{X_{t}}$ and assume $S_{t}$ is a martingale and $S_{0}=1$. For $t$ sufficiently small, by the monotonicity of the $\mathcal{L}^{p}$ norm, we have

$$
\mathbb{E}\left(S_{t}^{\frac{p_{1}}{t}}\right)^{\frac{t}{p_{1}}}<\mathbb{E}\left(S_{t}^{\frac{p_{2}}{t}}\right)^{\frac{t}{p_{2}}},
$$

or

$$
\mathbb{E}\left(S_{t}^{\frac{p_{1}}{t}}\right)<\mathbb{E}\left(S_{t}^{\frac{p_{2}}{t}}\right)^{\frac{p_{1}}{p_{2}}} .
$$

For $t$ sufficiently small, $\frac{p_{2}}{t}>1$, so by Jensen's inequality, we have

$$
\mathbb{E}\left(S_{t}^{\frac{p_{2}}{t}}\right) \geq \mathbb{E}\left(S_{t}\right)^{\frac{p_{2}}{t}}=1 .
$$

Thus

$$
\mathbb{E}\left(S_{t}^{\frac{p_{2}}{t}}\right)^{\frac{p_{1}}{p_{2}}} \leq \mathbb{E}\left(S_{t}^{\frac{p_{2}}{t}}\right),
$$

and we are done.

(ii) We now consider $-\infty<p_{2}<p_{1}<0$. We wish to prove that

$$
\mathbb{E}\left(e^{\frac{p_{1}}{t} X_{t}}\right) \leq \mathbb{E}\left(e^{\frac{p_{2}}{t} X_{t}}\right)
$$


for $t$ sufficiently small. For $t$ sufficiently small, by the monotonicity of the $\mathcal{L}^{p}$ norm, we have

$$
\mathbb{E}\left(\left(\frac{1}{S_{t}}\right)^{\frac{\left|p_{1}\right|}{t}}\right)^{\frac{t}{\left|p_{1}\right|}}<\mathbb{E}\left(\left(\frac{1}{S_{t}}\right)^{\frac{\left|p_{2}\right|}{t}}\right)^{\frac{t}{\left|p_{2}\right|}}
$$

or

$$
\mathbb{E}\left(\left(\frac{1}{S_{t}}\right)^{\frac{\left|p_{1}\right|}{t}}\right)<\mathbb{E}\left(\left(\frac{1}{S_{t}}\right)^{\frac{\left|p_{2}\right|}{t}}\right)^{\frac{\left|p_{1}\right|}{\left|p_{2}\right|}}
$$

For $t$ sufficiently small, $\frac{\left|p_{2}\right|}{t}>1$, so by Jensen's inequality, we have

$$
\mathbb{E}\left(\left(\frac{1}{S_{t}}\right)^{\frac{\left|p_{2}\right|}{t}}\right) \geq \mathbb{E}\left(\frac{1}{S_{t}}\right)^{\frac{\left|p_{2}\right|}{t}} \geq 1
$$

because $\frac{1}{S_{t}}$ is a submartingale. Thus

$$
\mathbb{E}\left(\left(\frac{1}{S_{t}}\right)^{\frac{\left|p_{2}\right|}{t}}\right)^{\frac{\left|p_{1}\right|}{\left|p_{2}\right|}} \leq \mathbb{E}\left(\left(\frac{1}{S_{t}}\right)^{\frac{\left|p_{2}\right|}{t}}\right),
$$

and we are done.

\section{Estimates for the standard normal distribution function}

Let $x>0$, and $n(z)=\frac{1}{\sqrt{2 \pi}} e^{-z^{2} / 2}$. Then we have the following estimate for $\Phi^{c}(x)=\int_{x}^{\infty} n(z) d z$

$$
\left(x+\frac{1}{x}\right)^{-1} n(x) \leq \Phi^{c}(x) \leq \frac{1}{x} n(x)
$$

(see section 14.8 in Williams [Will91]). 